infiltration of the surrounding tissues lead to stiffening and induration. Lnder treatment this diminishes, hence the increase noted in the mobility of the uterus. The improvement following the use of Doyen's serum has been ascribed to a similar effect.

The pelrio glands. - In four cases (Nos. 1, 4, 5, and 8) no glands could be found, despite a thorough search for them. Dr. J. H. Willett, who assisted me, bears me out in this. In Case 7 the operation was abandoned and the glands were not sought for. In the remaining five cases iliac glands were found on both sides in three (Cases 2, 3, and 10) and on one site in one (Case 9). In Case 6 the obturator glands on both sides were enlarged.

Microscopical examination has resulted as follows:-Case 2: no disease found on either side. Case 3: Right iliac glands cancerous. Case 9: Left iliac glands cancerous. Case 10: left iliac glands cancerous. Case 6: right obturator glands cancerous. Out of ten cases the glands were found to be cancerous in four-i.e., 40 per cent. It should be stated that sections of these glands were not taken by the serial method-the only trustworthy one-but merely from parts of the glands which appeared suspicious. Possibly, therefore, further investigation of Case 2 might result in the finding of diseased areas. Wertheim estimated that the glands were diseased in 35 per cent. of cases and his figures have been confirmed by Döderlein, Zweifel, and Kleinhaus. Von Rosthorn puts his figures as high as $57 \cdot 5$ per cent., while those of an American observer (Wakefield) are only from 25 to 30 per cent. Wertheim states that of 35 cases in 100 two-thirds show cancer cells arranged in alveoli. In the remaining third there was no alveolar arrangement, but instead tube or bag-shaped formations, lined by high cylindrical epithelium, often possessing cilia. $\mathrm{He}$ was inclined to believe that these appearances were to be regarded as having a connexion with carcinoma of the cervix until Meyer of Berlin found similar changes in the pelvic glands of patients who had died from eclampsia or peritonitis. Since this Wertheim has found the same appearances in glands from a patient whose death was attributed to multiple pelvic abscesses. His figures therefore require considerable modification.

Of my own cases, the most striking instances are Cases 6 and 10. Both were typical examples of cases suitable for vaginal hysterectomy. The disease appeared to be limited entirely to the cervix and the mobility of the uterus was normal in each case. Yet in Case 6 the right obturator gland was cancerous and from Case 10 was removed the largest cancerous gland I have yet seen. In both these cases the performance of vaginal hysterectomy would have been futile so far as eradication of the disease was concerned. Improvement succeeding removal of the primary growth would speedily have vanished as the secondary untouched focus advanced. Extirpation of cancerous glands is no guarantee that all disease has been eliminated. Experience may show that the presence of infected glands should be a contra-indication to radical operations, but in the present state of knowledge a "radical" operation, to deserve its title, should include removal of all possible areas which are commonly diseased. In four other cases (Nos. 2, 3, 4, and 7) the local conditions were such that vaginal hysterectomy would unhesitatingly have been performed. In only one of these the glands were found involved by cancer, so that out of six cases in which vaginal hysterectomy would ordinarily have been selected as the operation of choice the pelvic glands were cancerous in three. There were four cases (Nos. 1, 5, 8, and 9 ) in which the vaginal operation could not possibly have sufficed. Its performance would probably have been attempted in Case 1, but in the other three the interference with uterine mobility was too great even to justify the attempt. In three of these cases no glands were to be found at the operation (Nos. 1, 5, and 8).

Ligature of the internal iliac arteries produced no aftereffects at all beyond some temporary lividity of the skin area supplied by its branches. That it is not without its risks is shown by the case recorded by Cullen in which there occurred gangrene of the leg necessitating amputation. In the same case it was noted later that "it appeared as if all the pelvic organs were sloughing away." ligature of the artery is invaluable in some cases, absolutely checking the oozing of blood which obscures the field of a difficult operation and which thereby increases both its duration and its risks. After the ligature has been tightened the veins seem to collapse entirely; they were never tied for the reason that they were never seen. The only cases in which bæmor-
rhage was severe were those two (Nos. 4 and 7 ) in which venous plexust $s$ were wounded. The bleeding in Case 7 was alarmingly free but it was readily checked by pressure, as in a similar case related by Cullen.

Mortality. - Three deaths occurred out of ten cases, a rate of 30 per cent. It is scarcely necessary to recall the heavy mortality which led to the abandonment of Freund's operation-one of 76 per cent. Since 1895 this percentage has been steadily diminishing. Between 1900 and 1902 Wertheim operated on 31 cases with six deaths-18.2 per cent, Jonnesco's first series of ten cases had a mortality of 30 per cent.; his second series of 60 per cent.; and of the third series of ten cases every patient recovered. As he remarks, there seems to be a direct relation between the mortality on the one hand and experience in selecting cases and acquired operative skill on the other. This is perhaps, at present, the keynote of the matter. The operation, radical as it is and dangerous as it may be, requires that only selected cases be submitted to it-cases in which there is the possibility of removing the diseased uterus surrounded by a clear zone of sound, or apparently sound, tissues. Further desiderata are absence of much fat on the abdominal wall, a fairly roomy pelvis, and a good general condi. tion of health. The anatomical position and relations of the mamma and of the uterus differ too much to render any comparison between them of great value but there is a striking similarity between the histories of the surgical progress in the treatment of the cancerous affections of each. First, removal of part, then of the entire organ finally the organ plus its surrounding tissues, lymphatics, and glands. Extensive operations for cancer of the breast are practised now with but trifling mortality and are followed by more prolonged periods of subsequent immunity than were known in the past. Lessened mortality is largely due to modern asepsis but prolongation of immunity can only be due to freer excision. What has proved of value in the case of the cancerous mamma can at least be tried for the cancerous cervix uteri. Whilst routine removal by the vaginal operation ignores the possibility of outlring disease the results can only be disappointing. Is it not iime to face the facts, to recognise that in every 100 cases submitted to vaginal hysterectomy diseased areas remain untouched in at least a fourth of the number? Surely the procedure is only palliative in these cases, and as such should be limited in its applications.

It is possible that the clearing up of the mystery surrounding the etiology and pathology of cancer may establish a rational basis for treatment. It is also possible that the abdominal operation may prove to be inadequate. In the meantime my inclination is to regard the combined radical operation as the one which seems to offer a reasonable chance of success, provided that its application be restricted to suitable cases.

Liverpool.

\section{FIVE FATAL CASES OF ACUTE MENTAL DISORDER, PROBABLY ACUTE DELIRIUM.}

By EDWIN GOODALL, M.D., B S. LoND.,

$$
\text { F.R.C.P. LOND., }
$$

MEDICAL SUPERINTENDENT, JOINT COUYTIES ASYLUM, CARMARTHEN

The following cases appear to me to be worth recording on account of their rarity and severity, their rapid and lethal course, the obscurity of their pathogenesis, and, in three of them, the comparative insignificance of the naked-eye cerebral changes. As regards rarity, I find but five instances in a series of 451 necropsies, and undoubtedly th 3 very great majority of such cases come to necropsy. Having regard to our ignorance of the nature of the disorder which these cases illustrate, I am of opinion that all such should be brought forward with a view to stimulate inquiry. The notes in these cases are not so full as could be wished, for reasons beyond my control; nevertheless, they serve to give a fairly good picture of the conditions observed. In the record of post-mortem appearances only positive (morbid) conditions are noted for reason of space.

CASE 1.-The patient was a married woman, aged 43 years. This was her first attack and she was admitted 
to the asylum on May 21st, 1902. The family history, as given, was normal as regarded nervous disorders except that one sister suffered from "fits." The personal history was also normal. The patient had been healthy in mind and body until she got a severe attack of influenza at the close of December, 1901. Since that time she had not been quite well but was able to do her work up to a date two months prior to admission. No cause for the present attack was known apart from influenza. During the two months preceding admission she was low-spirited, lacrymose, and hypochondriacal. Recently she had been maniacal, with the usual symptoms. The following is a summary of the medical certificate of admission: "Lying in bed, shouting and muttering incoherently; mistakes of identity; some exaltation insomnia ; refusal of food, saying same was poisoned." On admission the patient was restless, resistive, hostile, and destructive; she was filthy in habits, noisy, unconscious of time and place, had delusions of persecution, hallucinations of vision, mistakes of identity, and much exaltation. Physically she was well nourished and powerful. Her face, hands, and feet were very cyanosed. The pulse was 88 , the respirations were 16 , and there was aortic regurgitation. There was tremor of the lips, the tongue, and the hands. Speech was tremulous. The pupils were equal and of normal contour there was no reaction to light and they were sluggish to accommodation. There were nebulæ about both corneæ. The knee-jerks were exaggerated. She was certified five days after admission by me as being in a state of "acute dementia, muttering unintelligibly, lying on the floor, moving constantly and aimlessly, resisting strongly, filthy in habits, destructive, refusing food. Physically well nourished and strong." The further history was as follows. She continued noisy, restless, and resistive. Sordes appeared on the lips. The day before death there were moist sounds over the lungs ; the pulse was quick and irregular; the temperature was not raised. She died on May 29th (eight days after admission)

Necropsy.-A post-mortem examination was held the next morning. The only morbid appearances were as follows. The dura mater was adherent to the skull cap in front ; there was slight opacity of the pia arachnoid over the sulci. The left ventricle was hypertrophied, the myocardium was friable and the aortic valves were thickened and atheromatous. The pharynx and trachea were very congested. The liver showed old nutmeg change. Nothing else abnormal was noted in the brain or elsewhere. The weight of the brain was (probably before stripping and unopened, but not stated) 48 ounces; the grey and white matter was of good consistence. The tangential fibres of the outermost layer of the cortex cerebri were found to be degenerated. A bloodserum tube inoculated with blood from the heart showed growth on the third day. A subculture from this tube was pronounced by Dr. D. N. Nabarro to be pure growth of the bacillus diphtheriæ.

CASE 2.-The patient was a single man, aged 21 years. It was his first attack and he was admitted to the asylum on Feb. 21st, 1905. The family history was as follows. The mother was epileptic and her father was "peculiar"; nothing further abnormal was ascertained. The patient's personal history was good. The present illness dated back to a recent attack of influenza. The present attack was of about one month's duration; the acuter phase had lasted one week. He had taken food badly; sleep was deficient; he had constipation, increasing incoherence, with hallucinations, and restlessness. The following is a summary of his medical certificate of admission: "Not a moment quiet; appears not to understand ; takes no notice ; incessant incoherent talk ; refusal of food ; insomnia." On admission his temperature was $101^{\circ} \mathrm{F}$. (thereafter it became normal, but on March 3rd it rose to $100.2^{\circ}$ and remained at between $99^{\circ}$ and $100^{\circ}$ until the morning of death when it was $101^{\circ}$ ). There was great physical reduction; the tongue was coated and tremulous ; there was marked constipation (great difficulty was experienced in overcoming this); and there were considerable rigidity and expression of pain on pressing the muscles. The knee-jerks were exaggerated. As regards mind, his attention could not be gained and he gave no indication of understanding, was unintelligible, and muttered unceasingly. He lay recumbent but was most restless and resistive. He could not control his emunctories. He was

1 See The Lancet, August 16th, 1902, p. 440. fed with great trouble. He was sleepless and appeared to be in pain. He got rapidly worse despite all treatment; he looked exceedingly ill and emaciated; the pulse becrme feeble; there was increasing dyspncea (there were a few râles at the bases of the lungs); he was sleepless and in constant motion. Death occurred on March 6th (13 days after admission, about six weeks after the illness was first noted).

Necropsy. - A post-mortem examination was made 41 bours after death. The only positive indications of disease were as follows. The dura mater was rather adherent to the skull; the leptomeninges were congested and the cerebral cortex was markedly so. The brain substance was softened. The weight of the brain (probably with soft membranes and before opening, though not stated) was 54 ounces. The lower lobes of both lungs were congested and there was a small recent pleuritic patch at the base of the right lung. The liver, the spleen, and the kidneys were congested.

CASE 3.-The patient was a married man, aged 40 years. This was his first attack. He was admitted to the asylum on April 15th, 1905. The family history was as follows. One grandfather was "eccentric" and one of the patient's children died from tuberculous meningitis ; nothing further abnormal could be ascertained. With regard to his personal history he drank too much at times-was " mad when in drink." The cause of the present attack (which was only of four days' duration) was great excitement during the religious revival. The following is a summary of the medical certificate of admission: "Religious mania with the usual symptoms, including refusal of food." $\mathrm{He}$ lived on milk-and-water for some time. On admission his temperature was subnormal (and it was not found above normal till the day of his death, when it was $100^{\circ} \mathrm{F}$. in the morning). He was emaciated, pale, and greatly reduced physically. The knee-jerks were much exaggerated. The month was dry, the tongue was coated and tremulous, there were sordes on the teeth, and constipation was present. Mentally the patient was very agitated, restless, shouting, gesticulating, and incoherent. He continued thus, passing evacuations under bim; he was fed by the tube, he was sleepless (no hypnotics were of any avail) Four days after admission he was removed to the infirmary ward owing to increasing exhaustion. $\mathrm{He}$ now took from the attendant four pints of milk and four eggs daily. His face was expressionless. The pulse was good. There was some dyspnœa with coarse râles at the base of the right lung. The temperature was normal. There was general muscular tremor. On the 28 nd he was restless, with increasing dyspnoea. The respirations were 45, the pulse was more feeble and rapid, and the temperature was $100^{\circ}$. He died at noon on April 22nd (seven days after admission). For the last three or four days of life he showed no mental reaction but lay in bed taking no notice, expressionless, silent, giving no indication of understanding what was said.

Necropsy.-A post-mortem examination (for an unavoidable reason) was made 71 hours after death. The only positive indications of disease were thickening of the pia arachnoid which peeled easily in large sheets; the grey and white matter of the brain was soft on section. (These last two conditions no doubt were aggravated by length of time between death and the necropsy.) There was congestion of both lungs; the spleen was congested and friable. There was nothing further. The weight of the brain was 53 ounces (with soft membranes).

CASE 4. - The patient was a married man, aged 46 years. It was his first attack. He was admitted to the asylum on Jan. 25th, 1896. His family history was as follows. His father was intemperate and an aunt had a stroke of hemiplegia. The patient was the father of nine children, of whom five died in youth from no special disease his wife had had two miscarriages also. With regard to his personal history he had had an attack of influenza three years before this illness but apparently made a good recovery. The present attack was of one week's duration ; the supposed cause was domestic trouble. The following is a summary of the medical certificate of admission: "Violence, some ideas of exaltation, refusal of food, restlessness." On admission he was very restless and resistive, unintelligible, agitated, required feeding by the tube, stared before him, and appeared to have visual hallucinations. He was utterly confused. Physically he was well nourished; he had an ulcer on the lower third of the right leg, on the antero-internal aspect, and several small scars about the front of the right knee, also one scar on the left knee. There was nothirg

$$
\text { c } 3
$$


else noteworthy except that the tongue was dry and furred. Subsequent entries note especially extreme restlessness"rolling about the floor"; the patient was only partially conscious. He was fed by the tube and the urine was drawn off. There were sordes on the lips and teeth. He got gradually feebler. On Feb. 3rd the pulse was 90 , scarcely perceptible, the temperature was $98^{\circ} \mathrm{F}$, the face and lips were dusky, and respiration was rapid. There was low muttering delirium. Death took place on Feb. 4th, ten days after admission.

Necropsy.-A post-mortem examination was held on Feb. 6th at noon. The skull cap was deeply injected and the dura mater was strongly adherent to either side of the middle line. The pia-arachnoid stripped normally, there were no erosions of the cortex, and the gyri were well arranged and of good size. All parts of the brain, including the grey and white matter, were extremely congested. There was no excess of cerebro-spinal fluid. Both lungs were congested. The brain weighed 58 ounces (probably with soft membranes). Nothing else was noteworthy. There was no evidence of syphilis in the testicles or elsewhere. This case during life was regarded as possibly one of dementia paralytica but there was nothing in the post-mortem appearances to indicate this disease.

CASE 5. - The patient was a married man, aged 51 years, a hosier. It was his first attack. He was admitted to the asylum on May 13th, 1905. His family history was free from disease of any importance. With regard to his personal history, he had had influenza "this year and many times." The cause of his present illness was believed to be religious ("revival") excitement; its duration was 14 days prior to admission. The following is a summary of the medical certificate of admission: " Religious mania, sleeplessness, restlessness." On admission the patient looked at least ten years older than he was; he was feeble and greatly emaciated. No cardiac murmur was heard; his pulse was feeble (120). The tongue was coated. He was very restless and resistive and was in a dazed condition. He refused food and was fed by the tube. He was quite incoherent. He passed evacuations beneath him. He remained in this condition until death occurred at 2.15 A.M. on the 17 th (four days after admission). On the 16th the morning temperature was $99 \cdot 4^{\circ}$ and the evening temperature was $100^{\circ}$.

Neoropsy.-A post-mortem examination was held on the 18 th at 1.45 P.M. Permission was given to examine the head only. Nothing abnormal was noted excepting that the pia arachnoid peeled in large sheets (not opaque), the brain tissue was soft and odematous, and the grey matter was but little congested. The weight of the brain with soft membranes was 55 ounces. This case resembled the others clinically. The necropsy was not, however, complete, as in the previous cases.

The above cases are, I think, best placed under the head. ing of "acute delirium" or "acute delirious mania." In three of them the marked congestion of the meninges and cerebrum and marked softening of the latter which are recorded in descriptions of acute delirium were not present. The brain-weights were, however, increased in all, pointing to œdema. The chief clinical features of these cases are, I think, the profound disturbance of cinsciousness, the restlessness (a constant movement, with resistance and without violence, the patient being recumbent), and the adynamic condition. This last rapidly increases and, in my experience, treatment is commonly of no avail. I believe that subcutaneous injections of sterilised salt solution should be tried more freely in these conditions, with the object of diluting and eliminating toxin. Hitherto I have not injected more than 500 cubic centimetres subcutaneously at a time, using Hayem's solution (seven grammes of common salt to one litre of water) every other day, sometimes daily, this being the method described by Jracquin, ${ }^{2}$ whose article first drew my attention to this treatment. Dr. Mosher of the Albany Hospital, New York, ${ }^{3}$ injects as much as 1700 cubic centimetres twice daily. Some prefer to introduce the solution into the bowel. I am not aware that there is sufficient experience to decide which method, if either, gives the better result. The first named is certainly the more trouble-ome, involving disinfection of apparatus.

In two of the above cases the mental illness was dated back by the friends to an attack of influenza and in two others there was intense religious excitement, both of which

2 Annales Médico-Psychologiques, Mas-June, 1900: Du Serum Arti ficiel en Psychiatrie.

ficird Report of the Department for Mental Disease, Albany Hospital, 1905 . are causes of acute delirium recognised by authors. The description given by the best English and foreign text-books of the condition above portrayed sufficiently demonstrates our lack of knowledge of its pathogenesis. Such descriptions commonly refer to the probability or certainty of toxæmia in acute delirium, even where the disease occurs independently of a specific malady, such as typhoid fever or pneumonia, but little or no information is forthcoming as to the nature of the toxin and as to its source beyond the statement that the latter may be exogenous or endogenous. As far as I am aware there is no record of the discovery of a specific organism in acute delirium apart from the observations of the Italian observers, Bianchi and Piccinino, who found a bacillus in the blood, an observation which is in need of ample confirmation. Cases of auto-intoxication with mental disturbance in diseases of the kidneys, the liver, the heart (cyanosis), and other organs have been described ${ }^{4}$ and the gastro-intestinal tract is commonly regarded and perhaps somewhat too familiarly referred to as a source of endogenous toxins, on which theory the stomach has been washed out and the intestines have been freely treated with such drugs as naphthalene with a view to disinfection, in the feasibility of which, as regards the bowel, I disbelieve. There is another form of auto-intoxication to which Mott has called attention ${ }^{5}$ and which consists in poisoning by the products of metabolism of nerve substance, such as choline. Choline, it appears from the source referred to, does not normally exist in the blood in sufficient quantities to be recognisable but can be detected in the blood "practically in any degenerative process affecting the nervous system if extensive enough." Its escape into the blood in large quantities and its accumulation therein may cause autointoxication. As, however, choline is not a powerful toxic substance (Mott found it in abundance in a case of disseminated sclerosis, which is certainly not a type of a sute toxic disease) it would appear improbable that this substance could produce such a rapid and fatal disease as acute delirium. But other products of the decomposition of the proteid matter of the nervous system may be more active poisons.

In diseases caused by exogenous toxins, such as the infectious fevers, there is as a rule a decided rise of temperature, whereas in cases of acute delirium the temperature is of ten but slightly raised, sometimes not at all. This point, together with the consideration that the latter disease is sometimes referable to a psychical cause with attendant physical reduction, would rather indicate that it is of the nature of an auto-intoxication. I consider that such cases as the above constitute one of many arguments for the establishment of a psychiatric department in connexion with our great teaching hospitals. For the conditions vecessary for research in the more obscure fields of psychological medicine, which conditions include proper facilities for animal experimentation and for collaboration with workers in allied departments of medical science, are not to be found, and in my belief will never be established, in county asylums in this country.

Carmarthen.

\section{THE DURATION OF LIFE AFTER THE APPEARANCE OF ALBUMINURIC RETINITIS.}

BY SIMEON SNELL, F.R.C.S. EDIN.,

OPHTHALMYC SURGEON, ROYAL INFIRMARY, SHEFFIELD; PROFESSOR OF OPHTHALMOLOGY, UNIVERSITY OF SHEFFIELD.

THE prognostic significance of albuminuric retinitis may well be regarded as a subject of considerable importance. Retinitis occurs in about 30 per cent. of all cases of Bright's disease. "This percentage is subjected to very slight variation by different observers. For instance, Norris stated that 25 per cent. of cases of nephritis were attended by retinal changes; Eales demonstrated by his statistics that its frequency was 28 per cent.; while Galezowski places it at 33 per cent. It seems to be more frequent in males than in females." 1

4 As, for example" by Jacobson, "Ueber Auto-intoxications-Psychosen," As, for example by Jacobson, "Ueber Auto-intoxication
Allgemeine Zeitschrift fir Pschiatrie, Band li., Heft 2.

3 Cromian Lectures, On the I egeneration of the Neurone 1900. Also Mociety.

1 Albuminuric Retinitis, by L. Webster Fox, A.M., M.D. New York Medical Journal, June $25 \mathrm{th}, 1904$. 